\title{
Study on Poloidal and Toroidal Electric Field Generations by Electron Cyclotron Heating in a Helical Plasma
}

\author{
Isao HIGASHI, Sadayoshi MURAKAMI \\ Department of Engineering, Kyoto University, Kyoto 6060-8501, Japan
}

(Received 28 November 2007 / Accepted 11 March 2008)

\begin{abstract}
Poloidal and toroidal electric fields generated by electron cyclotron heating are studied in a helical plasma. A linearized Fokker-Planck equation is solved by the adjoint method, assuming a helically symmetric configuration for simplicity. It is found that the poloidal and toroidal electric fields are generated near the bottom of the magnetic ripple, and that the larger radial flux is enhanced in a helical plasma compared with that in a tokamak plasma.
\end{abstract}

(C) 2008 The Japan Society of Plasma Science and Nuclear Fusion Research

Keywords: poloidal electric field, ECH, helical plasma

DOI: $10.1585 /$ pfr.3.S1079

\section{Introduction}

Electron cyclotron heating $(\mathrm{ECH})$ accelerates electrons perpendicularly, and generates trapped particles that tend to localize at the resonance region. These resonant trapped particles enhance an inhomogeneous electrostatic potential on a flux surface, and generate a poloidal electric field resulting in the large radial transport due to the radial drift by $\boldsymbol{E} \times \boldsymbol{B}$ drift.

Taguchi [1] evaluated the poloidal electric field generated by ECH in a tokamak plasma by solving an adjoint equation to the linearized Fokker-Planck equation with a quasi-linear diffusion term. Because of the deeper magnetic ripple due to the helical coils, a larger electric field would be generated in a helical plasma than that in a tokamak one.

In this paper, the poloidal and toroidal electric fields generated by ECH are investigated in a helical plasma in the collisionless regime, where the collsion frequency is much smaller than the bounce frequency of trapped electrons. Extending the method by Taguchi [1] to a three dimensional magnetic configuration, an equation for the electric field is derived. Then, the electric field is calculated assuming a helically symmetric configuration for simplicity. The electrostatic potentials are evaluated for the Large Helical Device (LHD) and the Helically Symmetric Experiment (HSX) using the realistic plasma parameters.

\section{Basic Equations}

In order to study the poloidal and toroidal electric fields in a helical plasma, we consider a magnetic configuration expressed in Boozer coordinates $(\psi, \theta, \zeta)$, where $\psi, \theta$ and $\zeta$ are the toroidal flux, poloidal angle, and toroidal angle, respectively. We assume that the $\mathrm{ECH}$ heating power is weak, and that the gyrophase- averaged distribution function for the electrons is slightly distorted from the Maxwell distribution function $f_{\mathrm{e} 0}(v)=$ $n_{\mathrm{e} 0}\left(m_{\mathrm{e}} / 2 \pi T_{\mathrm{e}}\right)^{3 / 2} \exp \left(-m_{\mathrm{e}} v^{2} / 2 T_{\mathrm{e}}\right)$, where $m_{\mathrm{e}}, T_{\mathrm{e}}$ and $n_{\mathrm{e} 0}$ are the electron mass, electron temperature, and electron density, respectively.

The distorted part $f_{\mathrm{e} 1}$ is determined by the following linearized drift kinetic equation:

$$
\begin{array}{r}
v_{\|} \boldsymbol{b} \cdot \nabla f_{\mathrm{e} 1}-C_{\mathrm{e}}\left(f_{\mathrm{e} 1}\right)=e v_{\|} \boldsymbol{E} \cdot \boldsymbol{b} \frac{\partial f_{\mathrm{e} 0}}{\partial W} \\
+Q_{\mathrm{rf}}\left(f_{\mathrm{e} 0}\right)-\frac{\partial f_{\mathrm{e} 0}}{\partial t}
\end{array}
$$

where the energy $W=\frac{1}{2} m v^{2}$ and the magnetic moment $\mu=v_{\perp}^{2} / 2 B$. Also, $\boldsymbol{b}=\boldsymbol{B} / B, v_{\|}=\boldsymbol{v} \cdot \boldsymbol{b}, v_{\perp}=\left(v^{2}-v_{\|}^{2}\right)^{1 / 2}, C_{\mathrm{e}}$ is the linearized Fokker-Planck collision operator, and $Q_{\mathrm{rf}}$ and $\boldsymbol{E}=-\nabla \phi$ are the velocity-space diffusion by the ECH and the electric field.

In order to calculate the electrostatic potential $\phi$, we introduce the adjoint equation,

$$
v_{\|} \boldsymbol{b} \cdot \nabla \tilde{f}_{m, n}+C_{\mathrm{e}}\left(\tilde{f}_{m, n}\right)=-\frac{v_{\mathrm{ee}} f_{\mathrm{e} 0} \mathrm{e}^{\mathrm{i}(m \theta+n \zeta)}}{\sqrt{g}}
$$

where $m$ and $n$ are the poloidal and toroidal mode numbers, respectively. Also, $v_{\mathrm{ee}}=\left(4 \pi n_{\mathrm{e} 0} e^{4} \ln \Lambda\right) / m_{\mathrm{e}}^{2} v_{\mathrm{e}}^{3}, v_{\mathrm{e}}=$ $\left(2 T_{\mathrm{e}} / m_{\mathrm{e}}\right)^{1 / 2}, \ln \Lambda$ is the Coulomb logarithm, and $\sqrt{g}=J$ is the Jacobian of the Boozer coordinates. We multiply Eq. (2) with $f_{\mathrm{e} 1} / f_{\mathrm{e} 0}$ and integrate over velocity space, and then take an average on the flux surface. Then, the electrostatic potential $\phi$ is given by

$$
\begin{aligned}
\frac{n_{\mathrm{e} 0} e}{T_{\mathrm{e}}} & \left\langle\frac{1}{\sqrt{g}} \mathrm{e}^{\mathrm{i}(m \theta+n \zeta)} \phi\right\rangle= \\
& -\frac{1}{v_{\mathrm{ee}}}\left\langle\int \frac{\tilde{f}_{m, n}}{f_{\mathrm{e} 0}}\left[Q_{\mathrm{rf}}\left(f_{\mathrm{e} 0}\right)-\frac{\partial f_{\mathrm{e} 0}}{\partial t}\right] \mathrm{d} \boldsymbol{v}\right\rangle \\
& +\left\langle\frac{n_{\mathrm{e} 1}}{\sqrt{g}} \mathrm{e}^{\mathrm{i}(m \theta+n \zeta)}\right\rangle
\end{aligned}
$$


where

$$
n_{\mathrm{e} 1} \equiv \int f_{\mathrm{e} 1} \mathrm{~d} \boldsymbol{v}
$$

Here, the flux surface average of a quantity $A,\langle A\rangle$, is

$$
\langle A\rangle=\oint \oint \mathrm{d} \theta \mathrm{d} \zeta \sqrt{g} A / \oint \oint \mathrm{d} \theta \mathrm{d} \zeta \sqrt{g}
$$

Also, we have used the relation

$$
\left\langle\int f v_{\|} \boldsymbol{b} \cdot \nabla g \mathrm{~d} \boldsymbol{v}\right\rangle=-\left\langle\int g v_{\|} \boldsymbol{b} \cdot \nabla f \mathrm{~d} \boldsymbol{v}\right\rangle,
$$

and the adjoint property of the collision operator,

$$
\int \frac{f_{\mathrm{e} 1}}{f_{\mathrm{e} 0}} C_{\mathrm{e}}\left(\tilde{f}_{m, n}\right) \mathrm{d} \boldsymbol{v}=\int \frac{\tilde{f}_{m, n}}{f_{\mathrm{e} 0}} C_{\mathrm{e}}\left(f_{\mathrm{e} 1}\right) \mathrm{d} \boldsymbol{v} .
$$

A similar relation can also be derived for the ions in a three dimensional configuration as follows:

$$
\frac{n_{\mathrm{i} 0} e Z_{\mathrm{i}}}{T_{\mathrm{i}}}\left\langle\frac{1}{\sqrt{g}} \mathrm{e}^{\mathrm{i}(m \theta+n \zeta)}\right\rangle=-\left\langle\frac{n_{\mathrm{i} 1}}{\sqrt{g}} \mathrm{e}^{\mathrm{i}(m \theta+n \zeta)}\right\rangle,
$$

where $Z_{\mathrm{i}}$ is the charge number of ions, and $T_{\mathrm{i}}$ is the ion temperature.

Expanding the electrostatic potential in a Fourier series we obtain

$$
\phi=\sum_{m, n=-\infty}^{\infty} \phi_{m, n} \mathrm{e}^{-\mathrm{i}(m \theta+n \zeta)} .
$$

The left hand side of Eq. (3) becomes

$$
\begin{aligned}
L H S & =\frac{n_{\mathrm{e} 0} e}{T_{\mathrm{e}}}\left\langle\frac{1}{\sqrt{g}} \phi_{m, n}\right\rangle \\
& =\frac{n_{\mathrm{e} 0} e}{T_{\mathrm{e}}} \frac{4 \pi^{2}}{\oint \oint \sqrt{g} \mathrm{~d} \theta \mathrm{d} \zeta} \phi_{m, n} .
\end{aligned}
$$

Using charge neutrality, the second term in the right-hand side of Eq. (3) becomes

$$
\begin{aligned}
\left\langle\frac{n_{\mathrm{e} 1}}{\sqrt{g}} \mathrm{e}^{\mathrm{i}(m \theta+n \zeta)}\right\rangle & =\left\langle\frac{Z_{\mathrm{i}} n_{\mathrm{i} 1}}{\sqrt{g}} \mathrm{e}^{\mathrm{i}(m \theta+n \zeta)}\right\rangle \\
& =-Z_{\mathrm{i}} \frac{n_{\mathrm{i} 0} e Z_{\mathrm{i}}}{T_{\mathrm{i}}}\left\langle\frac{1}{\sqrt{g}} \mathrm{e}^{\mathrm{i}(m \theta+n \zeta)} \phi\right\rangle \\
& =-\frac{n_{\mathrm{e} 0} e Z_{\mathrm{i}}}{T_{\mathrm{i}}} \frac{4 \pi^{2}}{\oint \oint \sqrt{g} \mathrm{~d} \theta \mathrm{d} \zeta} \phi_{m, n} .
\end{aligned}
$$

Transposing the second term in the right-hand side and arranging the equation, we obtain

$$
\begin{aligned}
& \frac{e \phi_{m, n}}{T_{\mathrm{e}}}=-\frac{v_{\mathrm{rf}}}{v_{\mathrm{ee}}} \frac{F_{m, n}}{1+Z_{\mathrm{i}} T_{\mathrm{e}} / T_{\mathrm{i}}} . \\
& F_{m, n}=\frac{\left\langle\int \frac{\tilde{f}_{m, n}}{f_{\mathrm{e} 0}}\left[Q_{\mathrm{rf}}\left(f_{\mathrm{e} 0}\right)-\frac{\partial f_{\mathrm{e} 0}}{\partial t}\right] \mathrm{d} v\right\rangle}{\left\langle\int \frac{W}{T_{\mathrm{e}}} Q_{\mathrm{rf}} \mathrm{d} v\right\rangle} \frac{\oint \oint \sqrt{g} \mathrm{~d} \theta \mathrm{d} \zeta}{4 \pi^{2}}
\end{aligned}
$$

where

$$
v_{\mathrm{rf}} \equiv \frac{1}{n_{\mathrm{e} 0} T_{\mathrm{e}}}\left\langle\int W Q_{\mathrm{rf}} \mathrm{d} v\right\rangle .
$$

\section{Solution of the Adjoint Equation}

Assuming the collisionless regime $v_{\mathrm{ee}}<<v_{\mathrm{b}}$, we can expand $\tilde{f}_{m, n}$ with a smallness parameter $\epsilon_{\mathrm{col}}=v_{\mathrm{ee}} / v_{\mathrm{b}}$ as $\tilde{f}_{m, n}=\tilde{f}_{m, n}^{(0)}+\tilde{f}_{m, n}^{(1)}+\cdots$, where $v_{\mathrm{b}}$ is the bounce frequency of the trapped electrons. Then the distribution functions $\tilde{f}_{m, n}^{(0)}$ and $\tilde{f}_{m, n}^{(1)}$ satisfy the equations for a three-dimensional configuration

$$
\begin{gathered}
v_{\|} \boldsymbol{b} \cdot \nabla \tilde{f}_{m, n}^{(0)}=0 \\
v_{\|} \boldsymbol{b} \cdot \tilde{f}_{m, n}^{(1)}+C_{\mathrm{e}}\left(\tilde{f}_{m, n}^{(0)}\right)=-\frac{v_{\mathrm{ee}} f_{\mathrm{e} 0}}{\sqrt{g}} \mathrm{e}^{\mathrm{i}(m \theta+n \zeta)} .
\end{gathered}
$$

The function $\tilde{f}_{m, n}^{(0)}$ is determined by the solubility condition for Eq. (13). We assume a linearized Fokker-Planck collision operation $C_{\mathrm{e}}$ as follows

$$
\begin{aligned}
C_{\mathrm{e}}(f) \approx\left(1+Z_{\mathrm{i}}\right) & \frac{v_{\mathrm{ee}}}{x^{3}} \frac{v_{\|}}{B} \frac{\partial}{\partial \mu}\left(\mu v_{\|} \frac{\partial f}{\partial \mu}\right) \\
& -f_{\mathrm{e} 0} \frac{v_{\mathrm{ee}}}{x^{2}} \frac{\partial}{\partial x}\left(\frac{f}{f_{\mathrm{e} 0}}\right),
\end{aligned}
$$

where $x=v / v_{\mathrm{e}}$. Then the solubility condition can be written as

$$
\begin{aligned}
2(1+ & \left.Z_{\mathrm{i}}\right) \frac{1}{x^{3}} \frac{\partial}{\partial \lambda}\left[\lambda\left\langle(1-\lambda B)^{1 / 2}\right\rangle_{b} \frac{\partial \tilde{f}_{m, n}^{(0)}}{\partial \lambda}\right] \\
& -\left\langle\frac{B}{(1-\lambda B)^{1 / 2}}\right\rangle_{b} \frac{f_{\mathrm{e} 0}}{x^{2}} \frac{\partial}{\partial x}\left(\frac{\tilde{f}_{m, n}^{(0)}}{f_{\mathrm{e} 0}}\right) \\
= & -\left\langle\frac{B}{(1-\lambda B)^{1 / 2}} \frac{1}{\sqrt{g}} \mathrm{e}^{\mathrm{i}(m \theta+n \zeta)}\right\rangle_{b} f_{\mathrm{e} 0},
\end{aligned}
$$

where $\lambda=2 \mu / v^{2}$, and the bounce average $\langle A\rangle_{b}$ is defined by

$$
\langle A\rangle_{b}=\left\{\begin{array}{lr}
\langle A\rangle & \left(0 \leq \lambda<\lambda_{c}\right) \\
\iint_{\lambda<1 / B} \sqrt{g} \mathrm{~d} \theta \mathrm{d} \zeta A / \oint \oint_{\left(\lambda_{c}<\lambda \leq \lambda_{\max }\right)}^{\sqrt{g} \mathrm{~d} \theta \mathrm{d} \zeta}
\end{array}\right.
$$

where $\lambda_{c}=1 / B_{\max } \lambda_{\max }=1 / B_{\min }$. We introduce the following eigenvalue equation to solve Eq. (15):

$$
\begin{aligned}
& \frac{\mathrm{d}}{\mathrm{d} \lambda}\left[\lambda\left\langle(1-\lambda B)^{1 / 2}\right\rangle_{b} \frac{\mathrm{d} G}{\mathrm{~d} \lambda}\right] \\
& +\frac{1}{2}\left\langle\frac{B}{(1-\lambda B)^{1 / 2}}\right\rangle_{b} \kappa G=0 \\
& \left(0 \leq \lambda<\lambda_{c}, \lambda_{c}<\lambda \leq \lambda_{\max }\right),
\end{aligned}
$$

where the boundary conditions are given by

$$
\begin{gathered}
G\left(\lambda_{c}-0\right)=G\left(\lambda_{c}+0\right) \\
\frac{\mathrm{d} G\left(\lambda_{c}-0\right)}{\mathrm{d} \lambda}=\frac{\mathrm{d} G\left(\lambda_{c}+0\right)}{\mathrm{d} \lambda} \\
G(0)=1, \quad \frac{\mathrm{d} G\left(\lambda_{\max }\right)}{\mathrm{d} \lambda} \text { is finite }
\end{gathered}
$$

The eigenfunctions $G_{j}$ with eigenvalues $\kappa_{j}$ satisfy the orthogonality condition

$$
\int_{0}^{\lambda_{\max }}\left\langle\frac{B}{(1-\lambda B)^{\frac{1}{2}}}\right\rangle_{b} G_{i} G_{j} \mathrm{~d} \lambda=0 \quad\left(\kappa_{i} \neq \kappa_{j}\right)
$$


Using this, we can express the solution of Eq. (16) in the form

$$
\tilde{f}_{m, n}^{(0)}=\sum_{j=1}^{\infty} \frac{x^{3}}{\left(1+Z_{\mathrm{i}}\right) \kappa_{j}+3} G_{j}(\lambda) S_{j} f_{\mathrm{e} 0},
$$

where $\kappa_{0}(=0)<\kappa_{1}<\kappa_{2}<\cdots$ and

$$
S_{j}=\frac{\int_{0}^{\lambda_{\max }} G_{j}\left\langle\frac{B}{(1-\lambda B)^{\frac{1}{2}}} \frac{1}{\sqrt{g}} \mathrm{e}^{\mathrm{i}(m \theta+n \zeta)}\right\rangle_{b} \mathrm{~d} \lambda}{\int_{0}^{\lambda_{\max }} G_{j}^{2}\left\langle\frac{B}{(1-\lambda B)^{\frac{1}{2}}}\right\rangle_{b} \mathrm{~d} \lambda} .
$$

Note that the eigenfunction $G_{0}=1$, so that $S_{0}=0$. Substituting the solution of Eq. (17) in Eq. (9), we obtain

$$
\begin{aligned}
F_{m, n}= & \frac{\oint \oint \sqrt{g} \mathrm{~d} \theta \mathrm{d} \zeta}{4 \pi^{2}} \frac{1}{\left\langle\int \frac{W}{T_{\mathrm{e}}} Q_{\mathrm{rf}} \mathrm{d} v\right\rangle} \\
& \times \sum_{j=1}^{\infty} \frac{S_{j}}{\left(1+Z_{\mathrm{i}}\right) \kappa_{j}+3}\left\langle\int x^{3} G_{j} Q_{\mathrm{rf}} \mathrm{d} v\right\rangle
\end{aligned}
$$

since

$$
\begin{aligned}
& \left\langle\int x^{3} G_{j} \frac{\partial f_{\mathrm{e} 0}}{\partial t} \mathrm{~d} v\right\rangle \\
\propto & \int_{0}^{\lambda_{\max }}\left\langle\frac{B}{(1-\lambda B)^{\frac{1}{2}}}\right\rangle_{b} G_{j} G_{0} \mathrm{~d} \lambda=0 \quad(n \geq 1) .
\end{aligned}
$$

\section{Evaluation of $\phi_{m, n}$}

\subsection{Quasi-linear diffusion by ECH}

We introduce the quasi-linear diffusion model for the velocity-space diffusions by ECH for evaluating the electrostatic potential. The quasi-linear diffusion term $Q_{\mathrm{rf}}$ is given by

$$
\begin{aligned}
Q_{\mathrm{RF}}=\frac{1}{v_{\perp}} & \frac{\partial}{\partial v_{\perp}}\left[D v_{\perp}\left(\frac{v_{\perp}}{v_{\mathrm{e}}}\right)^{2(l-1)}\right. \\
& \left.\times \delta\left(\omega-\frac{l \omega_{c}}{\gamma}-k_{\|} v_{\|}\right) \frac{\partial f_{\mathrm{e} 0}}{\partial v_{\perp}}\right]
\end{aligned}
$$

where $\gamma=\left[1-(v / c)^{2}\right]^{-\frac{1}{2}}, c$ is the speed of light in vacuum, and $l$ is the harmonic number. Also, $\omega$ and $\omega_{c}$ are the frequency of the injected wave and the non-relativistic electron-cyclotron frequency, respectively. In this paper, we only consider $\mathrm{X}$-mode wave heating, and $D$ does not depend on the velocity. Moreover, we introduce the relativistic resonance condition for simplicity as

$$
\omega-\frac{l \omega_{c}}{\gamma}-k_{\|} v_{\|} \approx \omega-l \omega_{c}\left(1-\frac{v^{2}}{2 c^{2}}\right)-k_{\|} v_{\|}=0 .
$$

This resonance condition becomes a semicircle in velocity space as follows:

$$
\left(\frac{v_{\|}}{v_{\mathrm{e}}}-\frac{1}{2 S}\right)^{2}+\left(\frac{v_{\perp}}{v_{\mathrm{e}}}\right)^{2}=\frac{1-4 u_{0} S}{(2 S)^{2}}
$$

where $u_{0}=\left(\omega-l \omega_{c}\right) / k_{\|} v_{\mathrm{e}}$ is the normalized parallel velocity of the resonant electrons, and $S=l \omega_{c} v_{\mathrm{e}} / k_{\|} c^{2}$ relates the strength of the relativistic correction. In the non-relativistic limit $S=0$, the resonance condition becomes the straight line $v_{\|} / v_{\mathrm{e}}=u_{0}$.

\subsection{Helically symmetric configuration}

It is very difficult to solve Eq. (18) in a real threedimensional configuration. Therefore, we assume a helically symmetric magnetic configuration for simplicity. The magnetic field strength of the helically symmetric configuration is given by

$$
\begin{aligned}
B & \left.=B_{0}\left\{1-\epsilon_{\mathrm{h}} \cos (M \theta+N \zeta)\right)\right\} \\
& =B_{0}\left(1-\epsilon_{\mathrm{h}} \cos \theta^{\prime}\right) \\
\theta^{\prime} & =M\left(\theta+\frac{N}{M} \zeta\right)
\end{aligned}
$$

where $M$ and $N$ are the poloidal and toroidal pitch numbers, and $\epsilon_{\mathrm{h}}$ is the strength of th helical ripple. Using $x=\lambda B_{\mathrm{h}}\left(1-\epsilon_{\mathrm{h}}\right)$, we rewrite Eq. (16) and the boundary condition as

$$
\begin{gathered}
x \alpha \frac{\mathrm{d}^{2}}{\mathrm{~d} x^{2}} G+\left(x \frac{\mathrm{d} \alpha}{\mathrm{d} x}+\alpha\right) \frac{\mathrm{d}}{\mathrm{d} x} G+\frac{1}{2} \beta \kappa G=0, \\
\alpha=\int_{\theta_{1}^{\prime}}^{\theta_{2}^{\prime}} \frac{1}{\left(1-\epsilon_{\mathrm{h}} \cos \theta^{\prime}\right)}\left(1-\frac{1-\epsilon_{\mathrm{h}} \cos \theta^{\prime}}{1-\epsilon_{\mathrm{h}}} x\right)^{1 / 2} \mathrm{~d} \theta^{\prime}, \\
\beta=\int_{\theta_{1}^{\prime}}^{\theta_{2}^{\prime}} \frac{1}{\left(1-\epsilon_{\mathrm{h}}\right)}\left(1-\frac{1-\epsilon_{\mathrm{h}} \cos \theta^{\prime}}{1-\epsilon_{\mathrm{h}}} x\right)^{-1 / 2} \mathrm{~d} \theta^{\prime}, \\
\frac{\mathrm{d} \alpha}{\mathrm{d} x}=-\frac{1}{2} \int_{\theta_{1}^{\prime}}^{\theta_{2}^{\prime}} \frac{1}{\left(1-\epsilon_{\mathrm{h}}\right)}\left(1-\frac{1-\epsilon_{\mathrm{h}} \cos \theta^{\prime}}{1-\epsilon_{\mathrm{h}}} x\right)^{-1 / 2} \mathrm{~d} \theta^{\prime}, \\
G \rightarrow \operatorname{const} \times\left[1-\frac{1}{2} \kappa(1-x)\right] \\
G \rightarrow 1-\frac{1}{2} \kappa x \\
x \rightarrow 0,
\end{gathered}
$$

where $\theta_{1}^{\prime}$ and $\theta_{2}^{\prime}$ are the turning points of trapped electrons.

\subsection{Results}

We calculate the eigenfunctions $G_{j}$ and eigenvalues $\kappa_{j}$ by a relaxation method in terms of Eq. (24). Before evaluating the electrostatic potential for the helical plasma, we analyze a tokamak plasma assuming the same magnetic field[1]: $B=B_{0} /(1+\epsilon \cos \theta)$, and evaluate the eigenfunctions and eigenvalues. The obtained result is in good agreement with that in the previous paper. We then calculate the eigenfunctions and eigenvalues for the helically symmetric configurations. The resulting eigenfunctions $G_{j}(j=1, . .5)$ are shown as a function of $\lambda B_{\mathrm{h}}\left(1-\epsilon_{\mathrm{h}}\right)$ for $\epsilon_{\mathrm{h}}=0.1$ and 0.3 in Fig. 1. The eigenvalues $\kappa_{n}$ are shown in Table 1. We can 

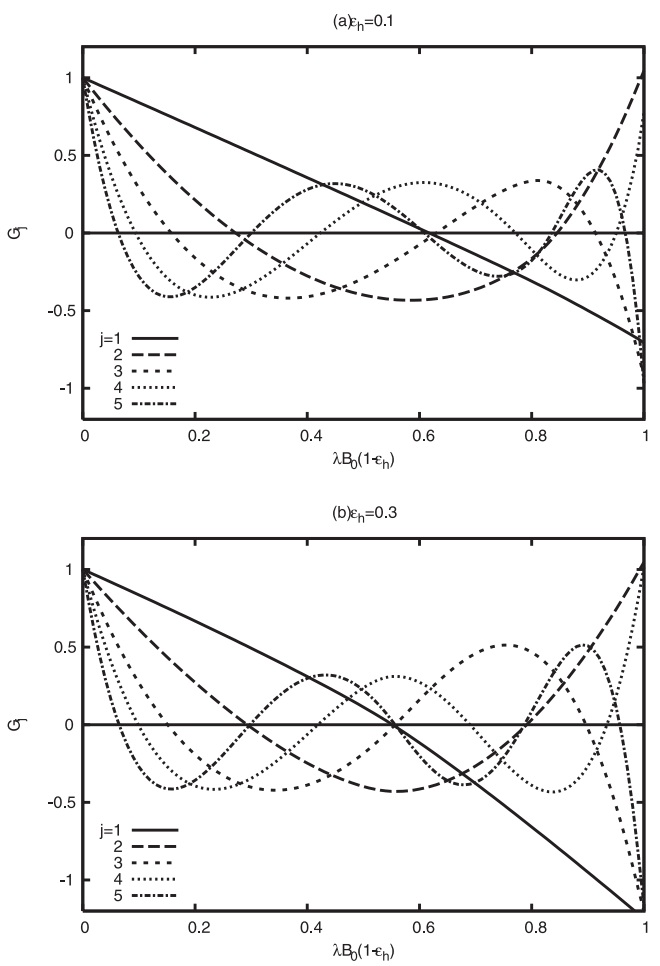

Fig. 1 Eigenfunctions $G_{j}(j=1, \ldots 5)$ as a function of $\lambda B_{\mathrm{h}}\left(1-\epsilon_{\mathrm{h}}\right)$ for the helical ripple $\epsilon_{\mathrm{h}}=0.1,0.3$.

Table 1 Eigenvalues $\kappa_{j}$ for a helically symmetric plasma.

\begin{tabular}{|c|ccccc|}
\hline$\epsilon_{\mathrm{h}} \backslash j$ & 1 & 2 & 3 & 4 & 5 \\
\hline 0.1 & 2.886 & 8.400 & 15417 & 26.643 & 40.196 \\
0.3 & 2.377 & 4.983 & 12.837 & 20.314 & 31.568 \\
\hline
\end{tabular}

see that the behavior of the eigenfunctions does not differ much from the tokamak ones, and that the difference of the eigenvalues is less than about $20 \%$.

Using the obtained eigenfunctions and eigenvalues for the helical symmetric configuration, we calculate the electrostatic potential from Eq. (9). In the calculation of Eq. (9), we take the summation up to $j=5$, and assume that RF waves are absorbed locally near $\theta^{\prime}=\theta_{0}$, i.e., $D \propto \delta\left(\theta^{\prime}-\theta_{0}\right)$. The electric field is represented as Eq. (9), and is analyzed in a helical plasma.

Figure 2 shows the $F_{m, n}$ values as a function of $\mathrm{S}$ in a helical plasma with $M=1$ and 2, changing the helical ripple $\epsilon_{\mathrm{h}} ; 0.1,0.3$. We plotted the results with different normalized parallel velocities; $u_{0}=0,0.5,-1.0 . F_{1,5}$ for $M=1$ helical plasma shows no clear difference from that for a tokamak plasma, whereas $F_{2,10}$ for $M=2$ helical plasma is about two times larger than that in the above two cases. This indicates that we would obtain a larger electrostatic potential as the poloidal pitch number increases. Figure 3 shows $F_{2,10}$ in $M=2$ helical plasma with $\theta_{0}=0$ and $u_{0}=0$, taking the summation from 1 to $J$, where $J=1$, $2,3,4,5,6,7$. The difference between $F_{2,10}$ with $J=5$ and $J=6$ is about $5 \%$ of $F_{2,10}$. Moreover, we found the
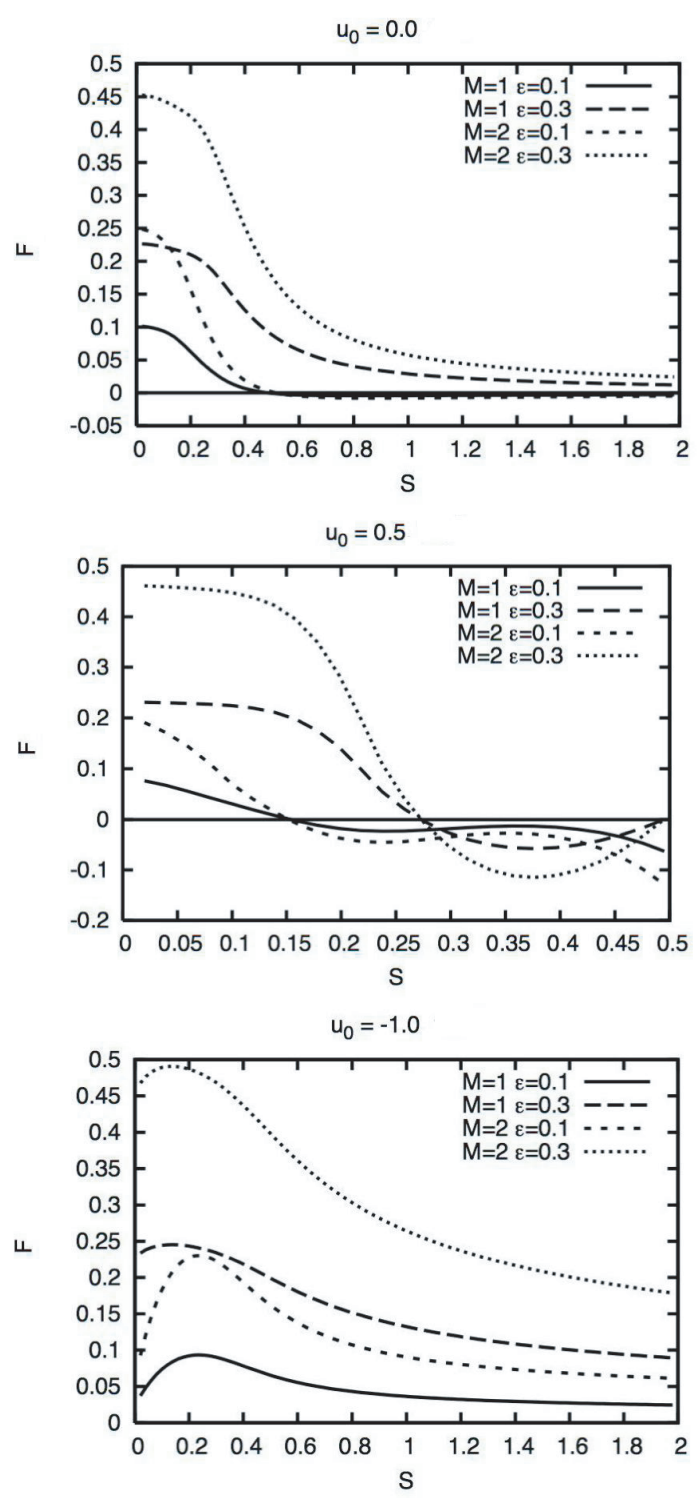

Fig. 2 Comparisons of $F_{m, n}$ in $M=1$ and $M=2$ helical plasma with $\theta_{0}=0$

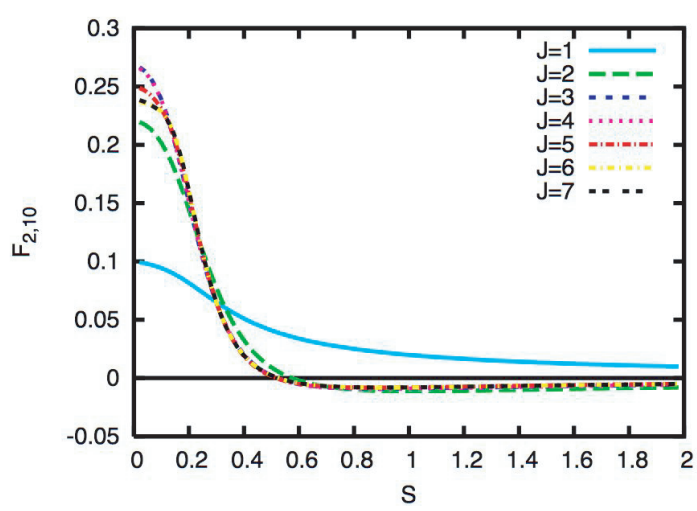

Fig. 3 Comparisons of $F_{2,10}$ in $M=2$ helical plasma with $u_{0}=0$ and $\theta_{0}=0$, taking the summation from 1 to $J(=1,2, \ldots, 7)$ 

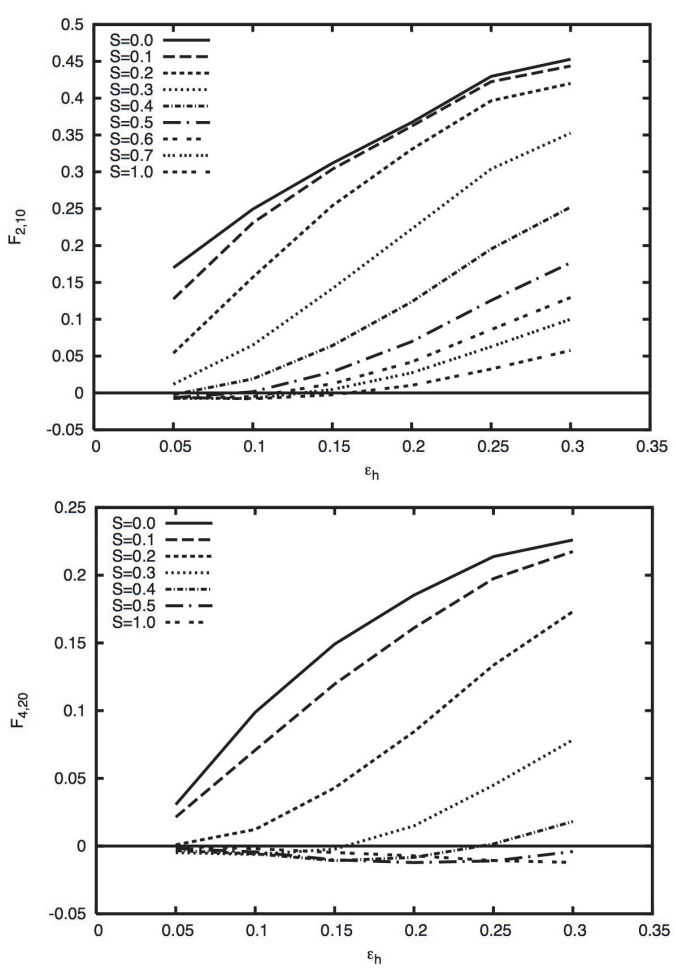

Fig. 4 Plots of $F$ as a function of $\epsilon_{\mathrm{h}}$ with $u_{0}=0$ and $\theta_{0}=0$.

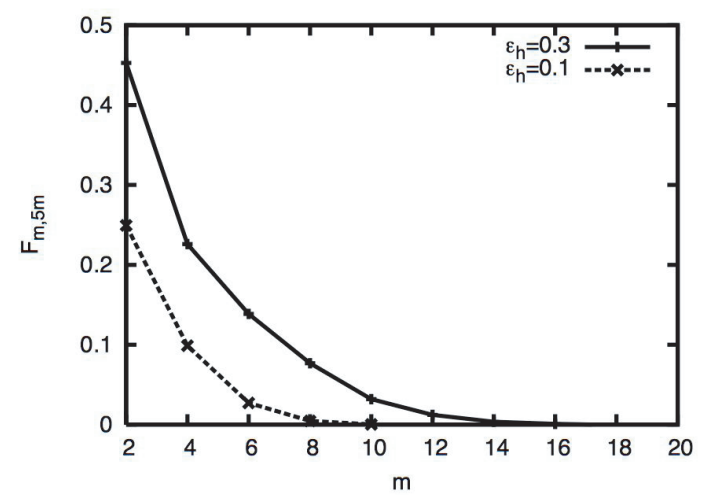

Fig. 5 Plots of $F$ as a function of the mode number $m$ for $S=$ $0, u_{0}=0$ and $\theta_{0}=0$. The helical ripple is chosen as $\epsilon_{\mathrm{h}}=$ $0.1,0.3$.

difference between $F_{2,10}$, considering that the summation up to $J=6$ and 7 is very small.

Next we study the helical ripple dependence of the electrostatic potential. Figure 4 shows $F_{2,10}$ and $F_{4,20}$ values as a function of $\epsilon_{\mathrm{h}}$ with $u_{0}=0$ and $\theta_{0}=0$, changing $S$ in a $M=2$ helical plasma. We can see monotonical growth of the $F$ value as $\epsilon_{\mathrm{h}}$ increases when $S$ is small. The $\epsilon_{\mathrm{h}}$ dependence of $F$ becomes small when $S$ reaches unity and the relativistic effect is important.

In order to evaluate the electrostatic potential, we have to sum up the values for various poloidal and toroidal modes. Figure 5 shows $F_{m, 5 m}$ values for various poloidal and toroidal modes in a $M=2$ helical plasma. We set

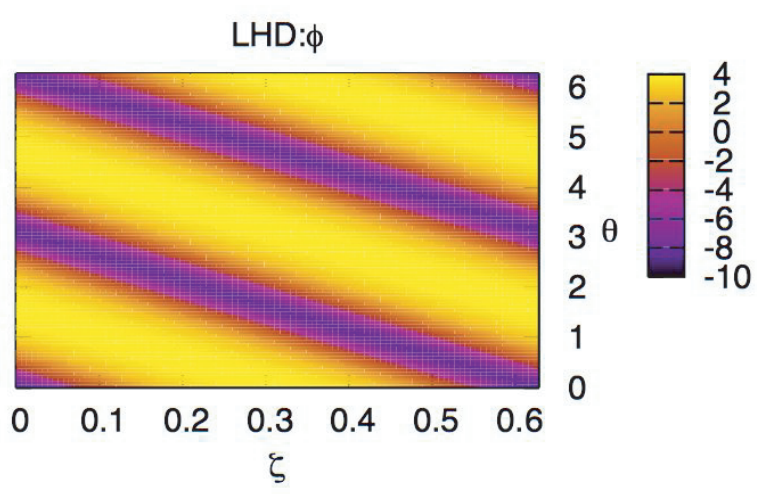

LHD: $E_{\theta}$

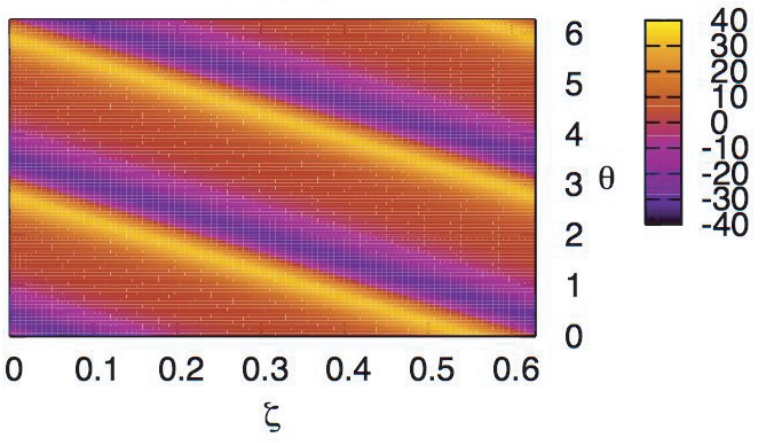

LHD: $E_{\zeta}$

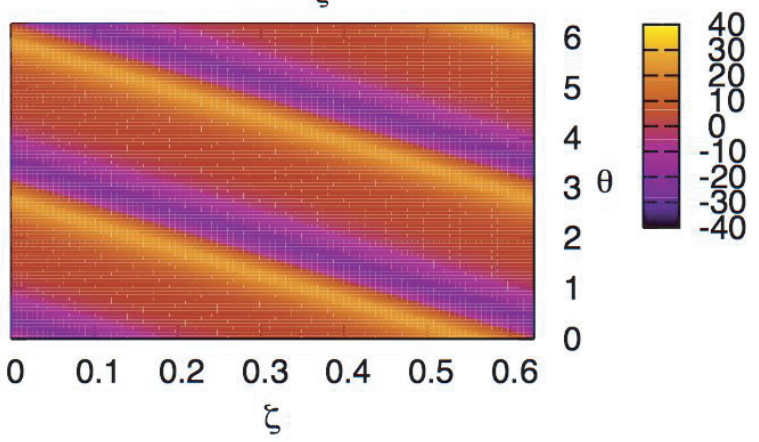

Fig. 6 The spatial distribution of the electrostatic potential and the generated electric field in LHD plasma.

$S=0$ and $u_{0}=0$. We can see that $F_{m, 5 m}$ decreases as the mode number increases, and the value is almost zero with $\mathrm{m}=14$.

Finally, we evaluate the electrostatic potential for the realistic plasma configuration of the LHD and HSX. In both devices, the ECH heating systems have been installed, and significant heating power is injected to start up and heat the plasma.

We assume the following plasma parameters for the LHD plasma: the density $n_{\mathrm{e}}=1.0 \times 10^{19} \mathrm{~m}^{-3}$, electron temperature $T_{\mathrm{e}}=3 \mathrm{keV}$, and ion temperature $T_{\mathrm{i}}=500 \mathrm{eV}$. The ECH heating power is set to be $1 \mathrm{~W} / \mathrm{cm}^{3}$. We also assume $S=0, u_{0}=0$, and $\theta_{0}=0$. The obtained spatial distribution of the electrostatic potential $\phi$ and the electric fields $E_{\theta}$ and $E_{\zeta}$ are shown in Fig. 6. We obtained the minimum value of the electrostatic potential as $\phi_{\min }=-8.454 \mathrm{~V}$, and the maximum values of poloidal and 

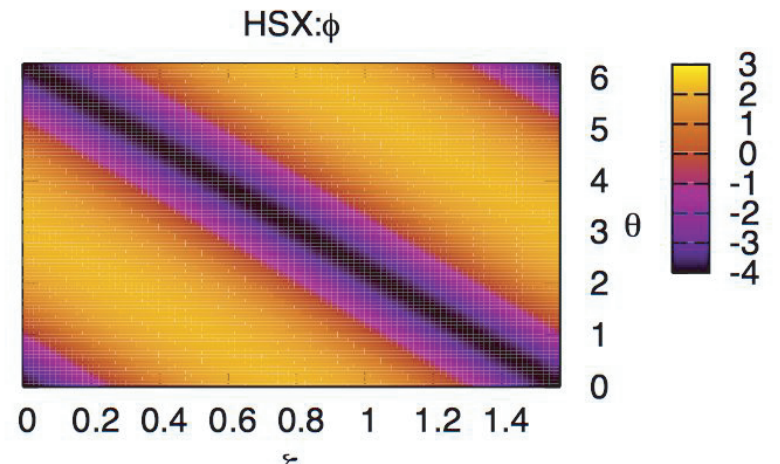

$\zeta$
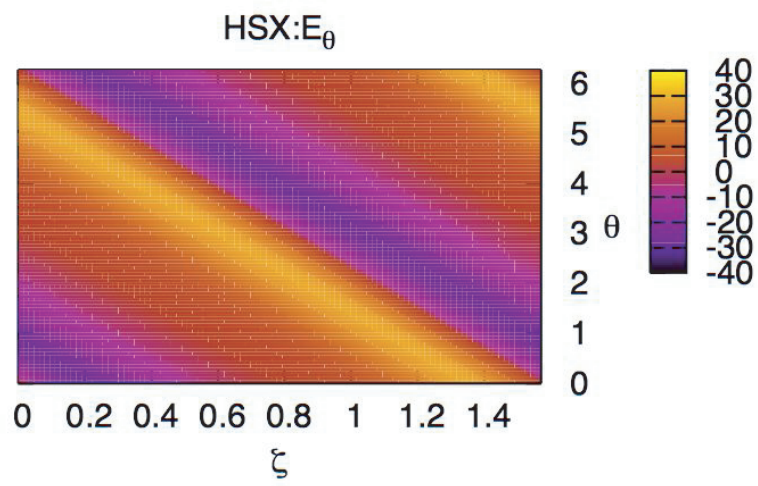

$H S X: E_{\zeta}$

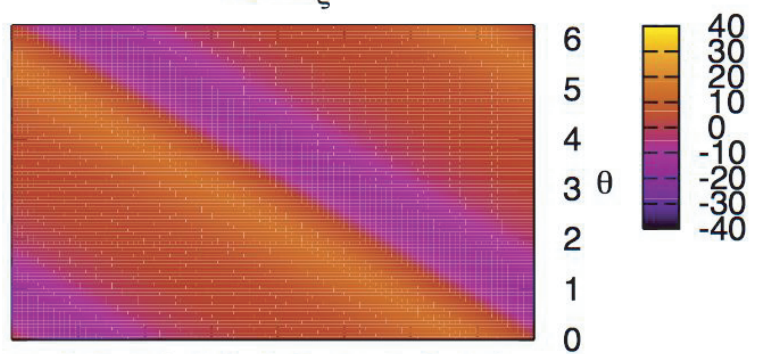

$\begin{array}{llllllll}0 & 0.2 & 0.4 & 0.6 & 0.8 & 1 & 1.2 & 1.4\end{array}$

$\zeta$

Fig. 7 The spatial distribution of the electrostatic potential and the generated electric field in HSX plasma.

toroidal electric fields as $E_{\theta, \max }=32.06 \mathrm{~V} / \mathrm{m}, E_{\zeta \max }=$ $24.66 \mathrm{~V} / \mathrm{m}$.

Next we evaluate the electrostatic potential in the HSX plasma. For the HSX, the plasma parameters are as follows: $n_{\mathrm{e}}=0.3 \times 10^{19} \mathrm{~m}^{-3}, T_{\mathrm{e}}=0.5 \mathrm{keV}$ and $T_{\mathrm{i}}=0.15 \mathrm{keV}$. The ECH heating power is also set to be $1 \mathrm{~W} / \mathrm{cm}^{3}$. We set $S=0, u_{0}=0$, and $\theta_{0}=0$. The obtained spatial distribution of the electrostatic potential $\phi$ and the electric fields $E_{\theta}$ and $E_{\zeta}$ are shown in Fig. 7. We obtained the minimum value of the electrostatic potential as $\phi_{\min }=-3.974 \mathrm{~V}$, and the maximum values of poloidal and toroidal electric fields as $E_{\theta, \max }=28.23 \mathrm{~V} / \mathrm{m}$, and $E_{\zeta, \max }=14.12 \mathrm{~V} / \mathrm{m}$, respectively.

We next compare the phase of the magnetic field ripple and the poloidal and toroidal electric fields in the LHD and HSX plasma to show the maximum point of the ExB radial flow. Figure 8 shows the amplitude of the magnetic
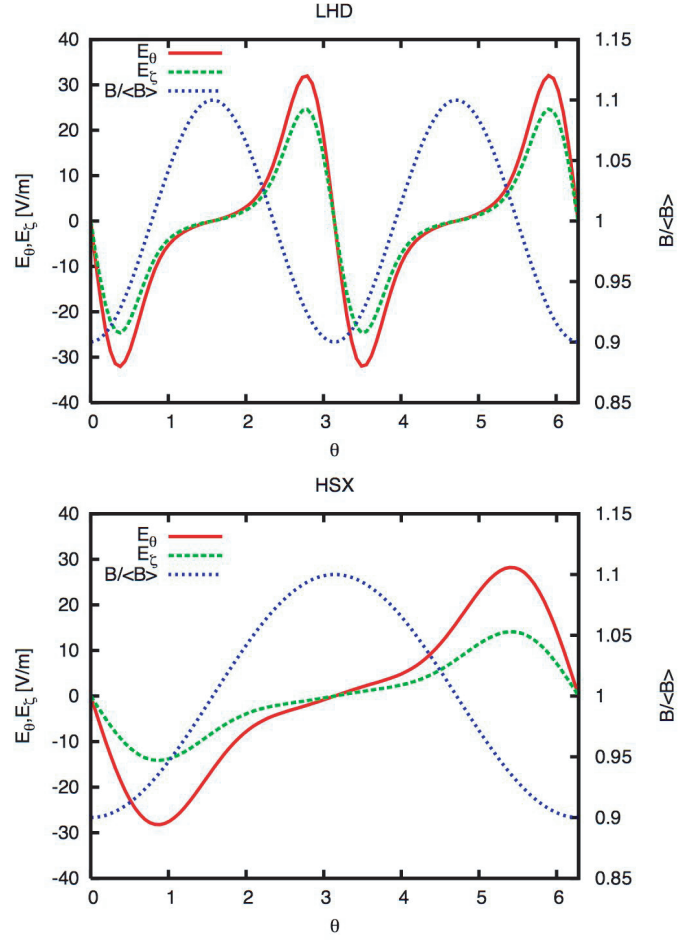

Fig. 8 Plots of the phase of $B /\langle B\rangle$ and $E_{\theta}$ at $\zeta=0$ as a function of the poloidal angle in the LHD and HSX plasma.

field and the poloidal and toroidal electric fields as a function of the poloidal angle. It is found that the maximum and minimum points of the poloidal electric field are near the bottom of the magnetic ripple.

This result shows that about two times larger minimum point of the electrostatic potential is generated in the LHD plasma than in the HSX plasma; however, the obtained poloidal electric field would be comparable due to the larger plasma size of LHD. Also, in the LHD plasma, the difference between the poloidal and toroidal electric field is small, but in the HSX plasma the poloidal electric field is two times larger than the toroidal electric field.

These potential values linearly depend on the ECH heating power, and we obtain a larger poloidal electric field with the stronger heating power.

\section{Conclusion}

We have studied the poloidal and toroidal electric fields generated by ECH in a helical plasma. We have extended the method by Taguchi [1] to a three-dimensional configuration, and the equation for the electrostatic potential has been derived. Then, the poloidal and toroidal electric fields have been calculated assuming a helically symmetric configuration for simplicity. We have found that the generated electric potential of the helical plasma in the LHD $(M=2)$ is about two times larger than that of the tokamak plasma. This indicates that the four times larger poloidal electric field is generated in a $M=2$ helical plasma. 
In this study, we have assumed a helically symmetric configuration instead of the realistic three-dimensional magnetic configuration. We will solve the adjoint equation Eq. (2) in the realistic magnetic configuration by modifying the GNET code [2], in which the linearized drift kinetic equation can be solved, and the results will be presented in the near future.

[1] M. Taguchi, J. Plasma Physics. 47, part 2, 261 (1992).

[2] S. Murakami et al., Nucl. Fusion 40, 693 (2000). 